\section{P191 (continued)}

teachers perceive a lack of consistency between home and school regarding behavioral, dietary, and social development expectations, teachers often think children need physical activity due to its importance for behavioral management and motor development rather than health, and teachers want children to eat healthy foods but may have limited nutrition knowledge and struggle with personal nutrition habits.

Conclusions and Implications: Childcare teachers have a prominent role in the development of many young children, although their importance in healthy lifestyle development is under-utilized. Teachers value nutrition and physical activity, but more so, from the child's development than personal health. Interventions to enhance healthy lifestyle habits in young children will need to incorporate the teacher perspective in order to maximize teacher cooperation in implementation and potential intervention impact.

Funding: None

\section{P192 SNAP-Ed Eligible Georgians' Experience Using an eLearning Nutrition Education Program - A Qualitative Study}

Sarah Stotz, MS, RD, LDN, CDE, sastotz@uga.edu, University of Georgia, 280 Dawson Hall, Athens, GA

30602; J. S. Lee, PhD, RD; J. Hibbs, EdD; A. White, MPH

Objective: The intent of this study was to understand experience of SNAP-Ed eligible Georgians regarding the use of a tailored eLearning nutrition education program, titled Food eTalk, in their free-living environment.

Design, Setting, and Participants: Focus group interviews were conducted in a statewide convenience sample of 45 SNAP-Ed eligible Georgians (age $37 \pm 14$ years, $48 \%$ black, 85\% with dependent children, 54\% receiving SNAP benefits). Participants engaged in two focus groups using a loaned smartphone/tablet with pre-paid data plan. Interviews focused on participants' experience with navigation, functionality, content, and thoughts on behavior change as a result of using Food eTalk.

Outcome Measures and Analysis: Focus group interviews were digitally recorded, transcribed verbatim, and coded to establish emergent themes using constant comparative method and Atlas.ti.

Results: Participants provided positive feedback regarding the ease of navigation of Food eTalk, entertaining interactive activities/games, and voiceover feature. They reported learning about sodium and food safety, and would have liked more information on diabetes, child feeding, and weight loss. Participants experienced various technical issues regarding functionality of Food eTalk. Barriers to translating eLearning to behavior change include the high cost of healthy foods, motivation to make healthy changes, preference for taste of 'unhealthy food', lack of time to prepare meals, and feeding picky children.

Conclusions and Implications: eLearning is a feasible and promising method to provide nutrition education to
SNAP-Ed eligible Georgians. Further technical development needs to address functionality concerns, and participant suggestions will be used to enhance and guide UGA SNAP-Ed eLearning programs.

Funding: Supplemental Nutrition Assistance Program Education, RNECE-South

\section{P193 The Perspective of the Learner and the Educator About a Food and Nutrition Education Program Developed With Women in Brazil}

Luiza Torquato, MPH, lu.torquato@gmail.com, University of Brasília, Darcy Ribeiro Campus, Faculty of Health Sciences (FS), Department of Nutrition, Observatory of Food Security and Nutrition Policies, Brasília-DF, Brazil, 70910-900; E. Recine, PhD

Objective: To analyze educators and learners' perceptions about the process of teaching and learning and the effectiveness of a food and nutrition education program developed with women.

Design, Setting, Participants, and Intervention: Case study with a qualitatively oriented approach carried out with 13 learners and 4 educators who participated in a community-based food and nutrition education program, developed for a low-income administrative region of the Federal District in Brazil. The program was conducted by a multi-professional team using active and participatory methods, a holistic approach to food and nutrition and following the principles and recommendations of the Dietary Guidelines for The Brazilian Population and The Reference Framework for Food and Nutrition Education.

Outcome Measures and Analysis: Semi-structured individual interviews were held, transcribed and analyzed using content analysis. The results were organized into categories, synthesized and interpreted.

Results: The program contributed to the awakening of a critical and holistic awareness in learners, enabling them to perceive themselves as protagonists of their reality and able to adopt a proactive attitude towards self-care (implementation of home gardens, fresh food and less processed food preferences, healthier cooking techniques) and transformation of the environment. Educators emphasized the importance of working with local communities, specifically diagnosis, sensitive listening, horizontal dialogue and of working in multi-professional team founded on collaboration, trust, and ethics to develop successful nutrition education programs.

Conclusions and Implications: Findings reveal a great potential of the adopted teaching-learning process and the program. Using active and participatory methods and a holistic approach to food may contribute to improving attitudes, self-efficacy and behaviors regarding eating habits, self-care and transformation of the environment.

Funding: National Council for Scientific and Technological Development, Ministry of Social Development, Fight Against Hunger of Brazil 